\title{
On Descriptions of Particle Transverse Momentum Spectra in High Energy Collisions
}

\author{
Fu-Hu Liu, Ya-Qin Gao, and Hua-Rong Wei \\ Institute of Theoretical Physics, Shanxi University, Taiyuan, Shanxi 030006, China \\ Correspondence should be addressed to Fu-Hu Liu; fuhuliu@163.com
}

Received 6 May 2014; Revised 20 July 2014; Accepted 14 August 2014; Published 28 August 2014

Academic Editor: Edward Sarkisyan-Grinbaum

Copyright ( $2014 \mathrm{Fu}-\mathrm{Hu}$ Liu et al. This is an open access article distributed under the Creative Commons Attribution License, which permits unrestricted use, distribution, and reproduction in any medium, provided the original work is properly cited. The publication of this article was funded by SCOAP ${ }^{3}$.

\begin{abstract}
The transverse momentum spectra obtained in the frame of an isotropic emission source are compared in terms of Tsallis, Boltzmann, Fermi-Dirac, and Bose-Einstein distributions and the Tsallis forms of the latter three standard distributions. It is obtained that, at a given set of parameters, the standard distributions show a narrower shape than their Tsallis forms which result in wide and/or multicomponent spectra with the Tsallis distribution in between. A comparison among the temperatures obtained from the distributions is made with a possible relation to the Boltzmann temperature. An example of the angular distributions of projectile fragments in nuclear collisions is given.
\end{abstract}

\section{Introduction}

In high energy collisions, one can use different distributions to describe the transverse momentum spectra of finalstate particles. For example, in the framework of a multisource thermal model which was proposed by us some years ago [1], one can use the Rayleigh distribution [26], Boltzmann distribution [7, 8], Fermi-Dirac distribution [9], and Erlang distribution [10,11] to describe the transverse momentum spectrum contributed by a given isotropic emission source. Particularly, in the classical version of the multisource thermal model, one can use the Rayleigh distribution to describe the transverse momentum spectrum, which is the same as that in the classical Maxwell ideal gas model. In the relativistic (and quantum) multisource thermal model, we can use the Boltzmann (or Fermi-Dirac or Bose-Einstein) distribution to describe the related spectrum.

In collisions at very high energy, it is important to find a proper distribution for the description of momenta or transverse momenta. Generally, the transverse momentum spectrum of final-state particles is a two-, three-, or multicomponent Boltzmann, Fermi-Dirac, Erlang, or other distributions. It shows that the types of sources produced in high energy collisions are two, three, or multiple. The rapidity spectrum of particles produced in the rest sources can be obtained by the spectrum of transverse momenta and assumption of isotropic emission, and the rapidity spectrum of final-state particles is the sum of different sources with different rapidity shifts [12].

In transverse momentum distribution, except for the contribution ratio, the temperature parameters are different for different components in the mentioned two-, three-, or multicomponent distributions. These two-, three-, or multicomponent distributions render temperature fluctuations in high energy collisions, which can be described by the nonextensive Tsallis distribution [13-22] or the Tsallis forms of the standard (Boltzmann, Fermi-Dirac, and BoseEinstein) distributions, and the temperature fluctuations can be described by a parameter (an entropic index) which characterizes the degree of nonequilibrium. In fact, the Tsallis distribution describes the final-state transverse momentum 
spectra by using two main parameters, a temperature $T$ and a nonequilibrium degree (or an entropic index) $q$, where $q>1$ and $q=1$ mean a nonequilibrium state and an equilibrium state, respectively [13-22]. The equilibrium indicates a thermal and kinetic equilibrium and does not require a chemical equilibrium.

In experiments at the Relativistic Heavy Ion Collider (RHIC) and the Large Hadron Collider (LHC) [23-28], the measured transverse momentum spectra are found to obey the Tsallis distribution [13-22] or similar distribution such as the Lévy distribution [11, 29-31], where the latter one is a special expression of the former one. The abundant data obtained at the RHIC and LHC have attracted many theoretical or modelling analyses [29-38], and some analyses show two or multiple processes' emission $[34,37]$. In some cases $[30,31]$, the Lévy distribution is also called the Tsallis distribution. The Tsallis distribution has more than one version used in literature [13-38], in which the power laws are somewhat different.

Different distributions can be adopted in the multisource thermal model [1]. Generally, for a given isotropic emission source, the temperature parameter should be a given value. In the case of using the same temperature parameter for different distributions, different results can be obtained. It is an important issue to choose a proper distribution for the rest sources in the multisource thermal model. In most cases, there is more than one distribution to be chosen. We are interested in studying the differences and similarities among these distributions of transverse momentum spectra. These differences and similarities are useful for the descriptions of the rest sources in the multisource thermal model [1].

In this paper, we compare the Tsallis, Boltzmann, FermiDirac, Bose-Einstein, Tsallis form of Boltzmann (Tsallis-B), Tsallis form of Fermi-Dirac (Tsallis-FD), and Tsallis form of Bose-Einstein (Tsallis-BE) distributions expected to be observed in high energy collisions. Following the formalism of these distributions in Section 2 which is based on [3844], a comparison and example are given in Sections 3 and 4 , respectively. Finally, we give our conclusions in Section 5.

\section{The Formalism}

The Tsallis distribution has more than one version [13-38]. In the present work, we adopt the version used in [38]. Correspondingly, the Tsallis forms of the three standard distributions have different expressions [39-44]. Based on [38], we can combine the different expressions for the given version to a uniform one. On the invariant particle momentum $(p)$ distribution, we have

$$
\begin{aligned}
& E \frac{d^{3} N}{d^{3} p} \\
& \quad=\frac{g V}{(2 \pi)^{3}} \sqrt{p^{2}+m_{0}^{2}} \\
& \quad \times\left\{\left[1 \pm \frac{q-1}{T}\left(\sqrt{p^{2}+m_{0}^{2}}-\mu\right)\right]^{ \pm(1 /(q-1))}+S\right\}^{-1},
\end{aligned}
$$

where $N$ is the particle number, $g$ is the degeneracy factor, and $V$ is the volume; $E, m_{0}$, and $\mu$ are the energy, the rest mass, and the chemical potential of the considered particle, respectively; $S=0,+1$, and -1 denote the Boltzmann, FermiDirac, and Bose-Einstein distributions, respectively; \pm sign is introduced in the above equation, + is for $\sqrt{p^{2}+m_{0}^{2}}>\mu$, and - is for $\sqrt{p^{2}+m_{0}^{2}} \leq \mu$ [38]. Then, the normalized Tsallis forms of the three standard distributions for momenta can be given by

$$
\begin{aligned}
f(p) & =\frac{1}{N} \frac{d N}{d p} \\
& =C_{1} p^{2}\left\{\left[1 \pm \frac{q-1}{T}\left(\sqrt{p^{2}+m_{0}^{2}}-\mu\right)\right]^{ \pm(1 /(q-1))}+S\right\}^{-1},
\end{aligned}
$$

where $C_{1}$ is the normalization constant. Further, we have the Tsallis forms of the three standard unit-density functions of transverse momentum $\left(p_{T}\right)$ and rapidity $(y)$ to be

$$
\begin{aligned}
& \frac{d^{2} N}{d y d p_{T}} \\
& =\frac{g V}{(2 \pi)^{2}} p_{T} \sqrt{p_{T}^{2}+m_{0}^{2}} \\
& \quad \times \cosh y\left\{\left[1 \pm \frac{q-1}{T}\right.\right. \\
& \left.\quad \times\left(\sqrt{p_{T}^{2}+m_{0}^{2}} \cosh y-\mu\right)\right]^{ \pm(1 /(q-1))} \\
& +S\}^{-1},
\end{aligned}
$$

where a \pm sign is introduced in the above equation, + is for $\sqrt{p_{T}^{2}+m_{0}^{2}} \cosh y>\mu$, and - is for $\sqrt{p_{T}^{2}+m_{0}^{2}} \cosh y \leq \mu$; the Tsallis forms of the three standard transverse momentum distributions are

$$
\begin{aligned}
& \frac{d N}{d p_{T}} \\
& =\frac{g V}{(2 \pi)^{2}} p_{T} \sqrt{p_{T}^{2}+m_{0}^{2}} \\
& \times \int_{y_{\min }}^{y_{\max }} \cosh y\left\{\left[1 \pm \frac{q-1}{T}\right.\right. \\
& \left.\times\left(\sqrt{p_{T}^{2}+m_{0}^{2}} \cosh y-\mu\right)\right]^{ \pm(1 /(q-1))} \\
& \quad+S\}^{-1} d y,
\end{aligned}
$$


where $y_{\max }$ and $y_{\min }$ denote the maximum and minimum rapidities, respectively; and the Tsallis forms of the three standard rapidity distributions are

$$
\begin{aligned}
& \frac{d N}{d y} \\
& =\frac{g V}{(2 \pi)^{2}} \cosh y \int_{0}^{p_{T \max }} p_{T} \sqrt{p_{T}^{2}+m_{0}^{2}} \\
& \quad \times\left\{\left[1 \pm \frac{q-1}{T}\left(\sqrt{p_{T}^{2}+m_{0}^{2}} \cosh y-\mu\right)\right]^{ \pm(1 /(q-1))}\right. \\
& \quad+S\}^{-1} d p_{T},
\end{aligned}
$$

where $p_{T \text { max }}$ denotes the maximum transverse momentum.

In particular, some special distributions can be obtained due to some limitations. According to [38], the Tsallis distribution which describes well experimental data of invariant particle momenta in high energy collisions can be given by

$$
\begin{aligned}
E \frac{d^{3} N}{d^{3} p}= & \frac{g V}{(2 \pi)^{3}} \sqrt{p^{2}+m_{0}^{2}} \\
& \times\left[1+\frac{q-1}{T}\left(\sqrt{p^{2}+m_{0}^{2}}-\mu\right)\right]^{-(q /(q-1))},
\end{aligned}
$$

where, comparing with (1), the limitations $\sqrt{p^{2}+m_{0}^{2}}>\mu$ and $S=0$ and the upper index $q /(q-1)$ are used in (6). Then, we have the normalized Tsallis distribution of momenta to be

$$
\begin{aligned}
f(p) & =\frac{1}{N} \frac{d N}{d p} \\
& =C_{2} p^{2}\left[1+\frac{q-1}{T}\left(\sqrt{p^{2}+m_{0}^{2}}-\mu\right)\right]^{-(q /(q-1))},
\end{aligned}
$$

where $C_{2}$ is the normalization constant. The unit-density function of $p_{T}$ and $y$ is [38]

$$
\begin{aligned}
& \frac{d^{2} N}{d y d p_{T}} \\
& \quad=\frac{g V}{(2 \pi)^{2}} p_{T} \sqrt{p_{T}^{2}+m_{0}^{2}} \\
& \quad \times \cosh y\left[1+\frac{q-1}{T}\left(\sqrt{p_{T}^{2}+m_{0}^{2}} \cosh y-\mu\right)\right]^{-(q /(q-1))} .
\end{aligned}
$$

Further, we have the transverse momentum distribution to be

$$
\begin{aligned}
\frac{d N}{d p_{T}}= & \frac{g V}{(2 \pi)^{2}} p_{T} \sqrt{p_{T}^{2}+m_{0}^{2}} \\
& \times \int_{y_{\min }}^{y_{\max }} \cosh y \\
& \times\left[1+\frac{q-1}{T}\right. \\
& \left.\times\left(\sqrt{p_{T}^{2}+m_{0}^{2}} \cosh y-\mu\right)\right]^{-(q /(q-1))} d y
\end{aligned}
$$

and the rapidity distribution to be

$$
\begin{aligned}
& \frac{d N}{d y} \\
& =\frac{g V}{(2 \pi)^{2}} \cosh y \\
& \quad \times \int_{0}^{p_{T \max }} p_{T} \sqrt{p_{T}^{2}+m_{0}^{2}} \\
& \quad \times\left[1+\frac{q-1}{T}\right. \\
& \left.\quad \times\left(\sqrt{p_{T}^{2}+m_{0}^{2}} \cosh y-\mu\right)\right]^{-(q /(q-1))} d p_{T} .
\end{aligned}
$$

Because $q$ is very close to 1 , we have the upper index $q /(q-1)$ in (6)-(10) to approximate $1 /(q-1)$. The upper index $1 /(q-1)$ is a result of a mean field approach [45] which gives a smaller $q$ too close to 1 .

Particularly, in the limit where $q \rightarrow 1$, the Tsallis distribution reduces to the standard Boltzmann distribution [38]. Considering the standard Fermi-Dirac distribution and the standard Bose-Einstein distribution, we uniformly have the three standard distributions to be

$$
\begin{aligned}
& E \frac{d^{3} N}{d^{3} p} \\
& \quad=\frac{g V}{(2 \pi)^{3}} \sqrt{p^{2}+m_{0}^{2}}\left[\exp \left(\frac{\sqrt{p^{2}+m_{0}^{2}}-\mu}{T}\right)+S\right]^{-1} .
\end{aligned}
$$

The normalized form of the three standard distributions for momenta can be given by $[46,47]$

$$
f(p)=\frac{1}{N} \frac{d N}{d p}=C_{3} p^{2}\left[\exp \left(\frac{\sqrt{p^{2}+m_{0}^{2}}-\mu}{T}\right)+S\right]^{-1},
$$


where $C_{3}$ is the normalization constant. The unit-density function of $p_{T}$ and $y$ is [38]

$$
\begin{aligned}
\frac{d^{2} N}{d y d p_{T}}= & \frac{g V}{(2 \pi)^{2}} p_{T} \sqrt{p_{T}^{2}+m_{0}^{2}} \\
& \times \cosh y\left[\exp \left(\frac{\sqrt{p_{T}^{2}+m_{0}^{2}} \cosh y-\mu}{T}\right)+S\right]^{-1}
\end{aligned}
$$

The transverse momentum distribution can be given by

$$
\begin{aligned}
\frac{d N}{d p_{T}}= & \frac{g V}{(2 \pi)^{2}} p_{T} \sqrt{p_{T}^{2}+m_{0}^{2}} \\
& \times \int_{y_{\min }}^{y_{\max }} \cosh y \\
& \times\left[\exp \left(\frac{\sqrt{p_{T}^{2}+m_{0}^{2}} \cosh y-\mu}{T}\right)+S\right]^{-1} d y .
\end{aligned}
$$

The rapidity distribution is

$$
\begin{aligned}
& \frac{d N}{d y} \\
& =\frac{g V}{(2 \pi)^{2}} \cosh y \\
& \quad \times \int_{0}^{p_{T \max }} p_{T} \sqrt{p_{T}^{2}+m_{0}^{2}} \\
& \quad \times\left[\exp \left(\frac{\sqrt{p_{T}^{2}+m_{0}^{2}} \cosh y-\mu}{T}\right)+S\right]^{-1} d p_{T} .
\end{aligned}
$$

In the above discussions, we have used the same temperature value in different distributions and obtained different results. For a given transverse momentum spectrum, different distributions can be used at different temperatures to give the same or similar results. The relations between the Tsallis temperature $T_{T}$ and Boltzmann temperature (physical temperature) $T_{B}$, Fermi-Dirac temperature $T_{\mathrm{FD}}$ and $T_{B}$, BoseEinstein temperature $T_{\mathrm{BE}}$ and $T_{B}$, Tsallis-B temperature $T_{\mathrm{TB}}$ and $T_{B}$, Tsallis-FD temperature $T_{\mathrm{TFD}}$ and $T_{B}$, and Tsallis-BE temperature $T_{\mathrm{TBE}}$ and $T_{B}$ are unclear. The simplest relations are approximately linear, especially for light and heavy flavor particles, respectively [37]. We have

$$
\begin{aligned}
T_{T} & =a_{T} T_{B}+(q-1) C_{T}, \\
T_{\mathrm{FD}} & =a_{\mathrm{FD}} T_{B}+C_{\mathrm{FD}}, \\
T_{\mathrm{BE}} & =a_{\mathrm{BE}} T_{B}+C_{\mathrm{BE}}, \\
T_{\mathrm{TB}} & =a_{\mathrm{TB}} T_{B}+(q-1) C_{\mathrm{TB}}, \\
T_{\mathrm{TFD}} & =a_{\mathrm{TFD}} T_{B}+(q-1) C_{\mathrm{TFD}}, \\
T_{\mathrm{TBE}} & =a_{\mathrm{TBE}} T_{B}+(q-1) C_{\mathrm{TBE}},
\end{aligned}
$$

respectively, where $a_{T, \mathrm{FD}, \mathrm{BE}, \mathrm{TB}, \mathrm{TFD}, \mathrm{TBE}}$ and $C_{T, \mathrm{FD}, \mathrm{BE}, \mathrm{TB}, \mathrm{TFD}, \mathrm{TBE}}$ are corrected factors in the relations. Naturally, we do not eliminate other possibilities on the relations.

We regard $T_{B}$ as the physical temperature due to the fact that $T_{B}$ can be obtained from the relativistic ideal gas model and it degenerates to the temperature in the classical ideal gas model. To give a detailed comparison for $T_{B}$ and other temperatures in experiments goes beyond the focus of the present work. The relations will be investigated in more detail using the data from RHIC and LHC. The above linear relations are expected to be approximately correct for light particles such as pions, kaons, and nucleons or they are expected to be approximately correct for heavy particles such as $J / \psi, \Lambda$, and $\Delta$. The slopes corresponding to light and heavy particles are different. For totally light and heavy particles, the relations seem to be two-linear [37].

\section{Comparison}

Before we give formally the figures, we have done some calculations for purposes of testing and checking. It is found that, in high energy collisions, the effect with $\mu \ll m_{0}$ can be neglected as we have known in a previous work [47]. Only in the case of $\mu \rightarrow m_{0}$, one can see some differences between quantum and nonquantum distributions. In the formal calculation, we take $m_{0}=138 \mathrm{MeV} / \mathrm{c}$ and $\mu=130 \mathrm{MeV}$ at a given temperature in the present work. According to the results of the thermal dynamic model [48], the critical temperature for phase transition from hadron matter to quark matter is $156 \mathrm{MeV}$. We take $T=160 \mathrm{MeV}$ for heavy ion collisions at the RHIC and LHC. In addition, we take $q=1.05,1.1,1.2$, and 1.3 for comparisons, which will make a large difference in distributions. The selected values of $q$ cover approximately possible range obtained from experimental data $[37,38]$.

Figure 1 presents the transverse momentum spectra calculated by the above several distributions. The solid curves, open circles, dashed and dotted curves which neared the open circles, open squares, and dashed and dotted curves which neared the open squares correspond to the Tsallis, Boltzmann, Fermi-Dirac, Bose-Einstein, Tsallis-B, Tsallis-FD, and Tsallis-BE distributions, respectively. In the calculation, we take $m_{0}=138 \mathrm{MeV} / \mathrm{c}, \mu=130 \mathrm{MeV}, T=160 \mathrm{MeV}$, and $q=1.05$ (Figure 1(a)), 1.1 (Figure 1(b)), 1.2 (Figure 1(c)), and 1.3 (Figure $1(\mathrm{~d})$ ), respectively, as discussed above. Figure 2 is 


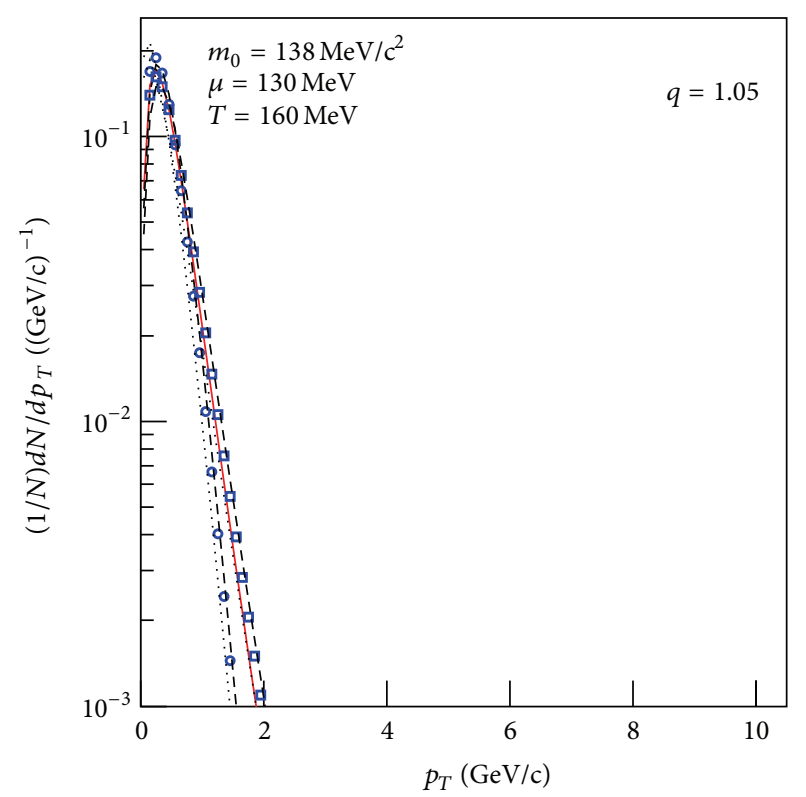

(a)

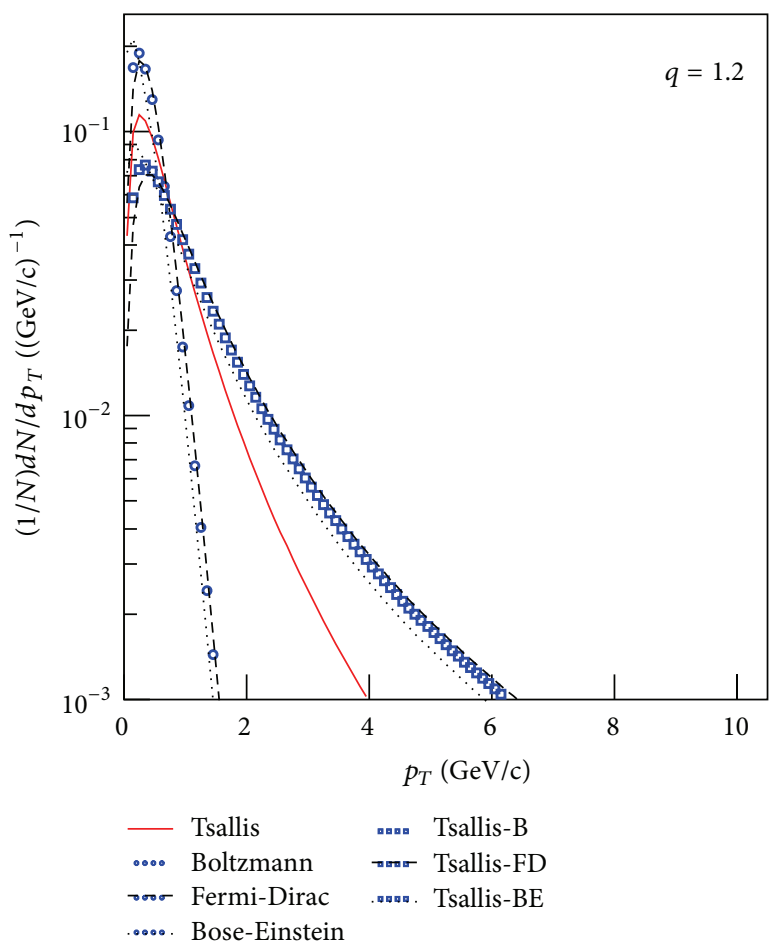

(c)

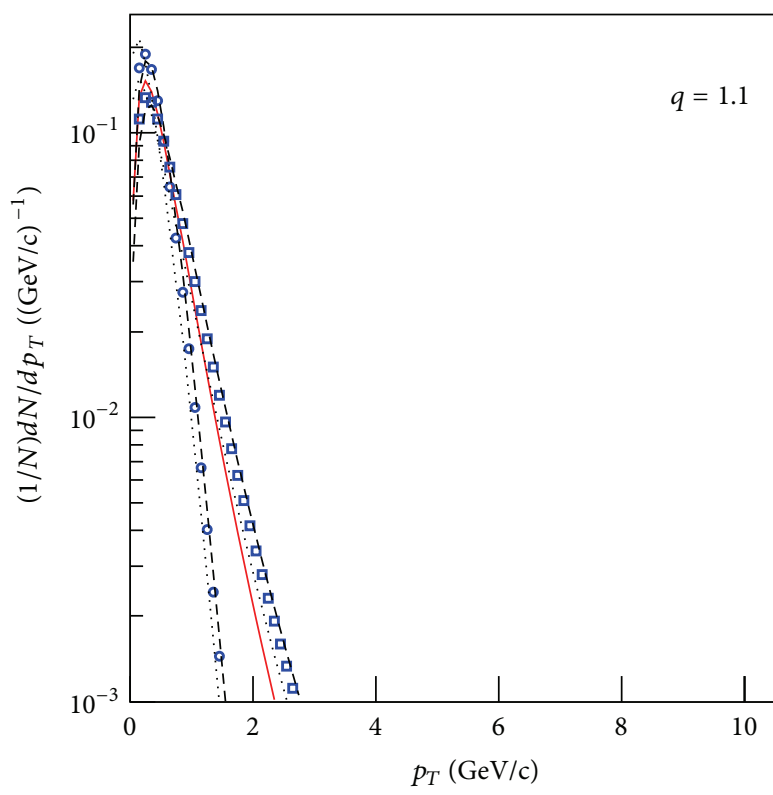

(b)

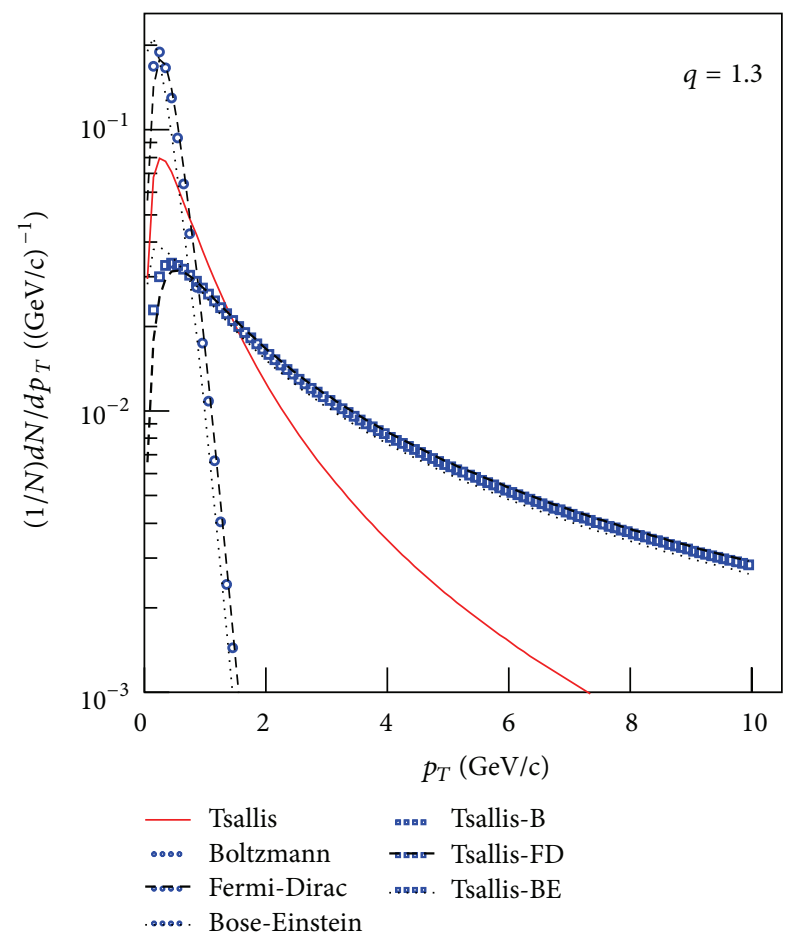

(d)

Figure 1: Transverse momentum spectra described by several distributions for $m_{0}=138 \mathrm{MeV} / \mathrm{c}, \mu=130 \mathrm{MeV}, T=160 \mathrm{MeV}$, and $q=1.05$ (a), 1.1 (b), 1.2 (c), and 1.3 (d), respectively. The solid curves, open circles, dashed and dotted curves which neared the open circles, open squares, and dashed and dotted curves which neared the open squares correspond to the Tsallis, Boltzmann, Fermi-Dirac, Bose-Einstein, Tsallis-B, Tsallis-FD, and Tsallis-BE distributions, respectively.

the same as that for Figure 1, but it shows the calculated results for rapidity spectra. (To check the validity of the numerical results in Figures 1 and 2, we have performed some additional Monte Carlo tests for the Tsallis distribution and found that the two results are in agreement with each other).
One can see from Figures 1 and 2 that, at a given temperature, the three standard distributions result in narrow spectra; the Tsallis-B, Tsallis-FD, and Tsallis-BE distributions result in wide spectra; and the Tsallis distribution results belong to the middle spectra. The quantum effect, that is, the difference in 


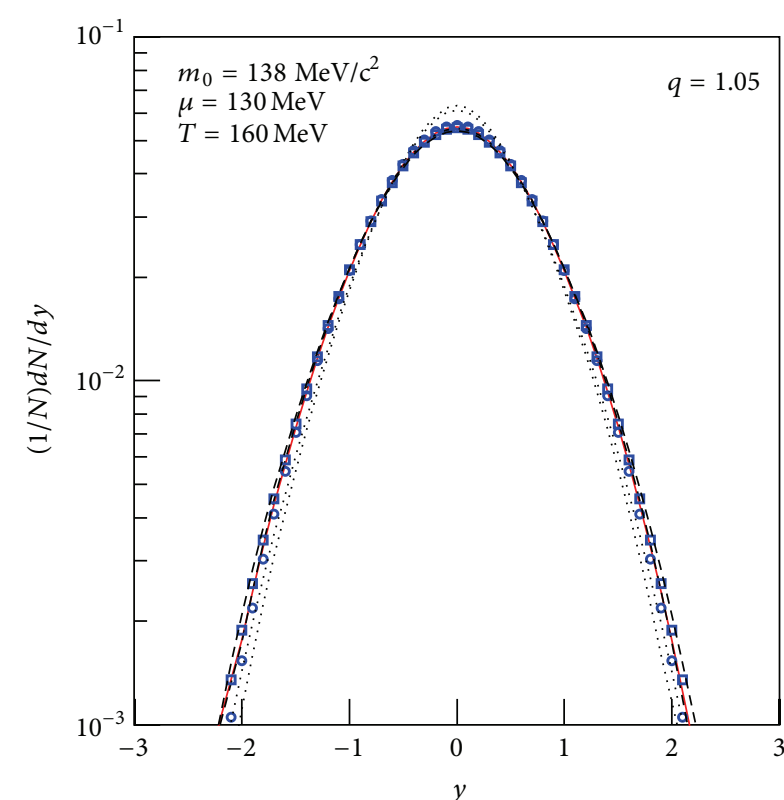

(a)

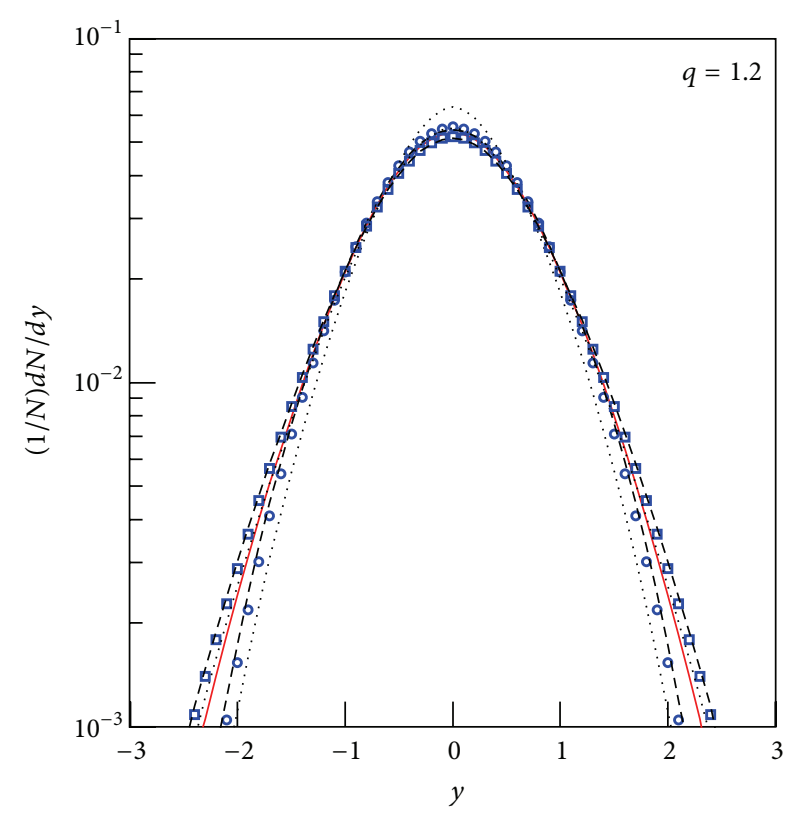

(c)

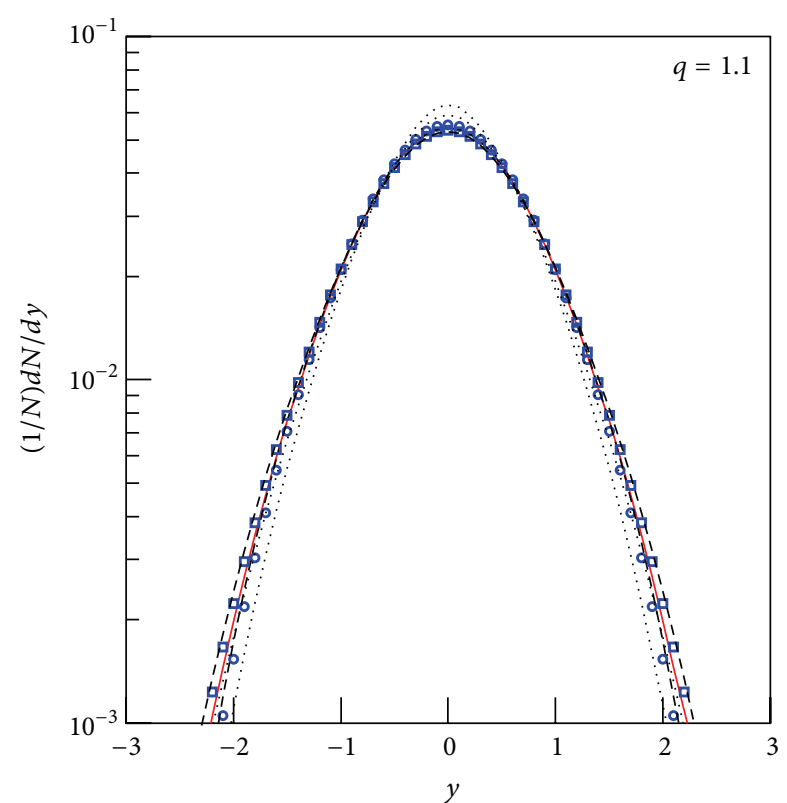

(b)

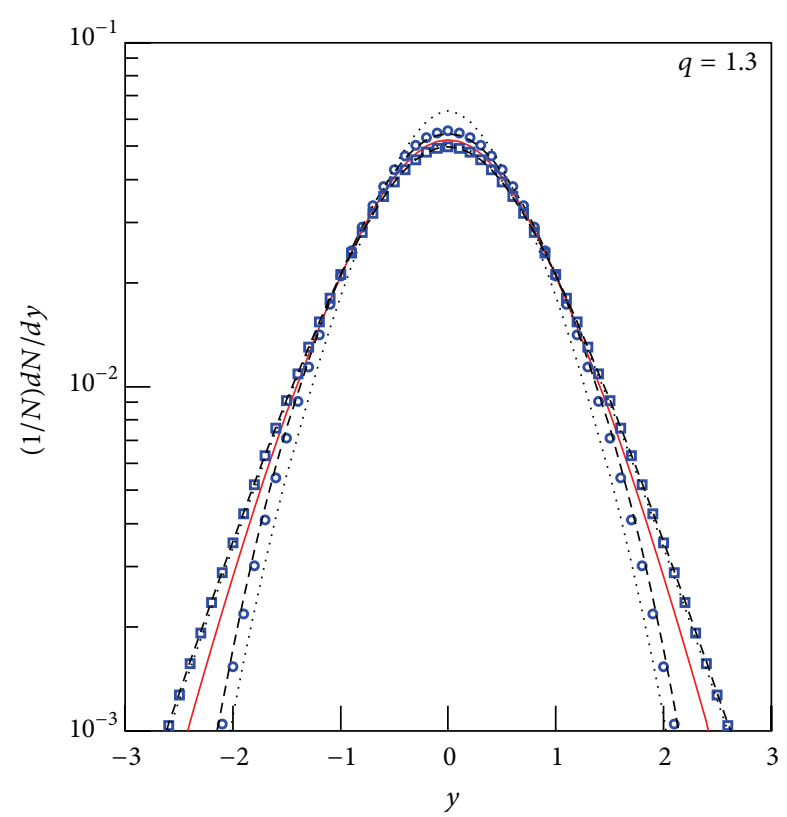

(d)

FIgURE 2: It is the same as for Figure 1, but it shows the rapidity spectra.

the results for different $S$, can be observed in the case of using the given set of parameters. One can also expect that the twoor three-temperature standard distributions fit approximately the Tsallis distribution, and the multitemperature standard distribution and the two- or three-temperature Tsallis distribution fit approximately the Tsallis forms of the standard distributions. Particularly, if the Tsallis distribution describes the temperature fluctuations in wide transverse momentum spectrum which can be fitted by a two- or three-component Boltzmann distribution, the Tsallis forms of the standard distributions describe large temperature fluctuations in wider transverse momentum spectrum which can be fitted by a multicomponent Boltzmann distribution.

In fact, our previous work [49] shows that one can use the multisource thermal model [1] to describe the transverse momentum spectra of particles produced in soft process in proton-proton and nucleus-nucleus collisions at RHIC and LHC energies. The standard Boltzmann distribution can be used to describe the particle behavior for single source. Then, the experimental transverse momentum spectrum is 


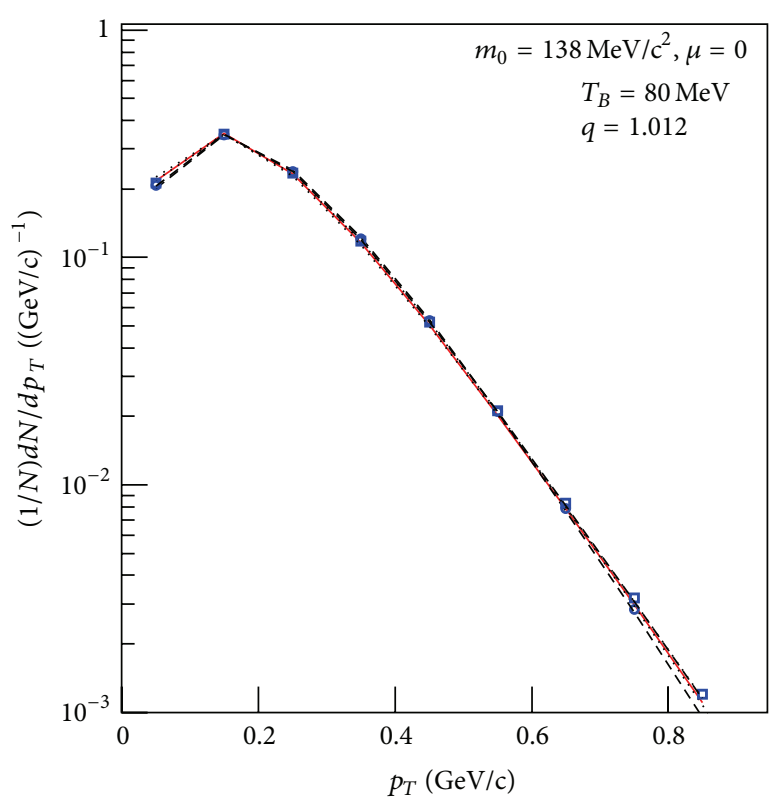

(a)

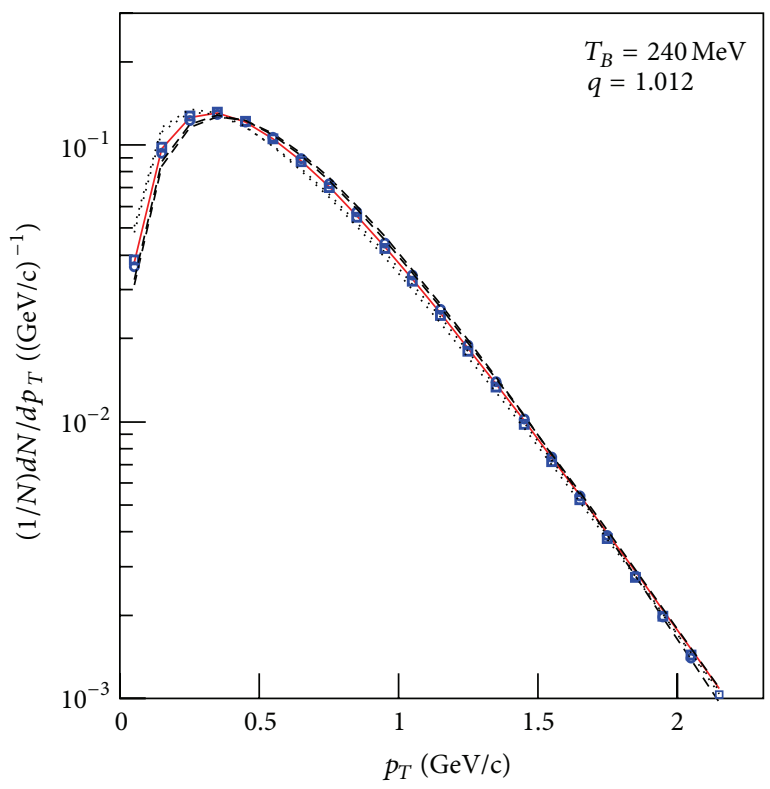

(c)

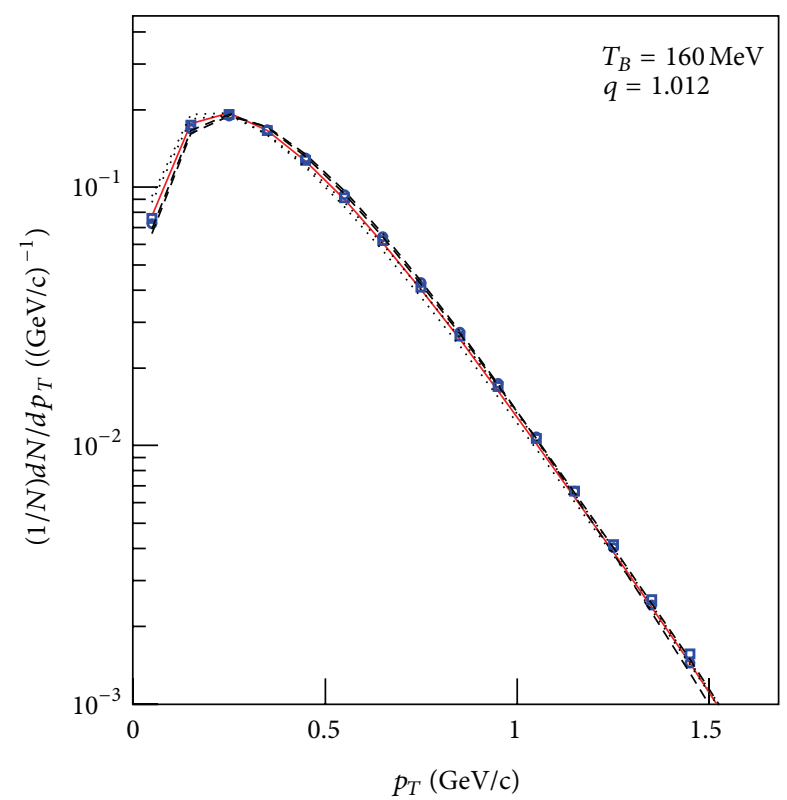

(b)

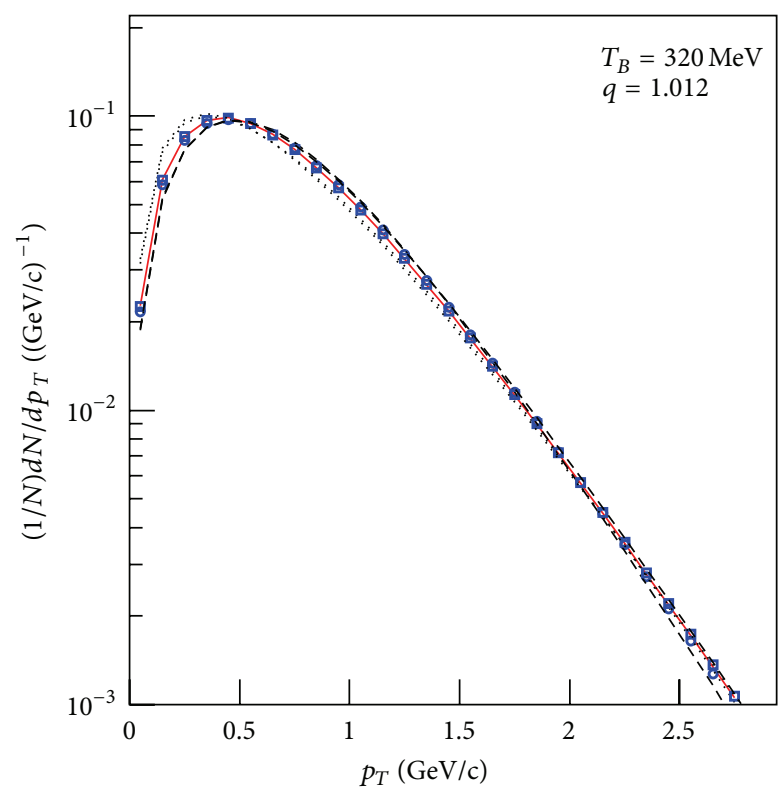

(d)

Figure 3: Comparisons of transverse momentum spectra for different distributions. Figures 3(a)-3(d) correspond to different $T_{B}$ indicated in the panels and a given $q$ of 1.012. The meanings of the curves and symbols are the same as those for Figure 1.

described by single, two-, or three-component Boltzmann distribution. If we consider the hard process which results in a wider transverse momentum spectrum, the multicomponent Boltzmann distribution is needed. In [37] and by us in [22], the Tsallis distribution is shown to describe successfully the transverse momentum (mass) spectra in high energy collisions. One can use the Tsallis distribution to describe uniformly the single, two-, and three-component Boltzmann distributions. A multicomponent Boltzmann distribution describes a wider transverse momentum spectrum which can be fitted by the Tsallis forms of the standard distributions or by a two- or three-component Tsallis distribution.

Figure 3 shows the comparisons of different distributions with Boltzmann distribution on the same or similar transverse momentum spectrum. In the comparisons, we take $T_{B}=80$ (Figure 3(a)), 160 (Figure 3(b)), 240 (Figure 3(c)), and $320 \mathrm{MeV}$ (Figure 3(d)), respectively; and $m_{0}=138 \mathrm{MeV} / \mathrm{c}^{2}$, $\mu=0$, and $q=1.012$ for the four cases. The meanings of different curves and symbols are the same as those for Figure 1. To give the same or similar result as the Boltzmann 
TABLE 1: Values of parameters corresponding to different distributions in Figure 3. The temperatures are in the units of MeV.

\begin{tabular}{lccccccc}
\hline Figure & $T_{B}$ & $T_{T}$ & $T_{\mathrm{FD}}$ & $T_{\mathrm{BE}}$ & $T_{\mathrm{TB}}$ & $T_{\mathrm{TFD}}$ & $T_{\mathrm{TBE}}$ \\
\hline Figure 3(a) & 80 & 75 & 79 & 81 & 75 & 74 & 75 \\
Figure 3(b) & 160 & 150 & 156 & 163 & 150 & 147 & 1.012 \\
Figure 3(c) & 240 & 229 & 236 & 247 & 224 & 223 & 1.012 \\
Figure 3(d) & 320 & 305 & 310 & 330 & 302 & 298 & 310 \\
\hline
\end{tabular}

distribution, the selected temperatures for other distributions are listed in Table 1. One can see that, for the same or similar transverse momentum distribution, comparing with the Boltzmann temperature (physical temperature), the standard Bose-Einstein distribution measures a high temperature, and other distributions measure low temperatures, for a small value of $q$. It is difficult to get the same or similar results by different distributions with proper temperatures for a large $q$.

Different temperatures extracted from the same or similar transverse momentum spectrum result in different thermodynamics and statistics. The most final-state particles are fermions, and the Fermi-Dirac statistics is expected to be the most appropriate description for single source. In high energy collisions, the quantum effect is small and the Boltzmann distribution is expected to be the most appropriate refill. Different thermodynamics and statistics give different expressions of transverse momentum spectra, which extract different temperatures. For a given system, the present work shows that the temperature found from Boltzmann distribution is smaller when compared with that from BoseEinstein distribution and is larger when compared with those from other distributions.

Although comparing the distributions to some data in proton-proton, proton-nucleus, or nucleus-nucleus collisions would lead to some interesting conclusions and we have not compared the present results with experimental data [23-28], the experimental groundwork of the present work is reliable. In fact, we have used the single or two-component Boltzmann distribution and the Tsallis distributions to describe experimental data in our previous work [22, 49-51]. The extracted temperatures are in the range used in the present work. For a narrow spectrum, all the distributions are very similar for a specific choice of temperatures and a small value of entropic index. We can conclude that all the distributions are equally good to describe the narrow spectrum. Because high energy collisions contain abundant contents such as entropy density [52], phase transition [53], and flow effects [54], more careful and broad investigations are needed in future.

\section{An Example: The Angular Distribution of Projectile Fragments in Nuclear Collisions}

In the above discussions, we have used the rest frame of the considered source and studied the behaviors of particles produced in violent interacting region such as the participant part in nucleus-nucleus collisions. In fact, these formulas can be also used in nonviolent interacting region such as the spectator parts in nucleus-nucleus collisions. As an example, we study here the space angular distributions of projectile fragments in fixed target experiments in the laboratory reference frame.

Let $p_{\text {beam }}, A_{F}$, and $\Theta$ denote the beam momentum per nucleon, the nucleon number of a considered projectile fragment, and the emission angle of the projectile fragment, respectively. The momentum per nucleon of the projectile fragment is assumed to be $p_{\text {beam. }}$. We have the transverse momentum of the projectile fragment to be $p_{T}=$ $A_{F} p_{\text {beam }} \sin \Theta$. Because $p_{T}$ can obey the distributions discussed in Section 2, we have the $\Theta$ distributions as in the following.

The Tsallis forms of the three standard distributions for the emission angles of projectile fragments are

$$
\begin{aligned}
& \frac{d N}{d \Theta}=F(\Theta) \\
& \quad \times \int_{y_{\min }}^{y_{\max }} \cosh y \\
& \quad \times\left\{\left[1 \pm \frac{q-1}{T}\right.\right. \\
& \quad \times\left(A_{F} \sqrt{p_{\text {beam }}^{2} \sin ^{2} \Theta+m_{N}^{2}}\right. \\
& \quad \times \cosh y-\mu)]^{ \pm(1 /(q-1))} \\
& +S\}^{-1} d y,
\end{aligned}
$$

where

$$
F(\Theta)=\frac{g V \sin 2 \Theta}{2(2 \pi)^{2}} A_{F}^{3} p_{\text {beam }}^{2} \sqrt{p_{\text {beam }}^{2} \sin ^{2} \Theta+m_{N}^{2}}
$$

and $m_{N}$ denotes the rest mass of a nucleon; a \pm sign is introduced in the above equation, + is for $A_{F} \sqrt{p_{\text {beam }}^{2} \sin ^{2} \Theta+m_{N}^{2}} \cosh y>\mu$, and - is for $A_{F} \sqrt{p_{\text {beam }}^{2} \sin ^{2} \Theta+m_{N}^{2}} \cosh y \leq \mu$. The Tsallis distribution 
for the emission angles of projectile fragments can be given by

$$
\begin{aligned}
& \frac{d N}{d \Theta} \\
& =F(\Theta) \\
& \quad \times \int_{y_{\min }}^{y_{\max }} \cosh y \\
& \quad \times\left[1+\frac{q-1}{T}\left(A_{F} \sqrt{p_{\text {beam }}^{2} \sin ^{2} \Theta+m_{N}^{2}}\right.\right. \\
& \quad \times \cosh y-\mu)]^{-(q /(q-1))} d y,
\end{aligned}
$$

where, comparing with (22), the limitations $A_{F} \sqrt{p_{\text {beam }}^{2} \sin ^{2} \Theta+m_{N}^{2}} \cosh y>\mu$ and $S=0$ and the upper index $q /(q-1)$ are used in (24). One can also use the upper index $1 /(q-1)$ in (24). The three standard distributions for the emission angles of projectile fragments are

$$
\begin{aligned}
& \frac{d N}{d \Theta}= \\
& \quad \times \int_{y_{\min }}^{y_{\max }} \cosh y \\
& \quad \times\left[\exp \left(\frac{A_{F} \sqrt{p_{\text {beam }}^{2} \sin ^{2} \Theta+m_{N}^{2}} \cosh y-\mu}{T}\right)\right. \\
& \quad+S]^{-1} d y
\end{aligned}
$$

where $S=0$ or +1 for projectile fragments.

Particularly, in the whole phase space, the classical ideal gas model gives the probability distribution of emission angles for the projectile fragments to be

$$
\frac{1}{N} \frac{d N}{d \Theta}=\frac{A_{F} p_{\text {beam }}^{2} \sin 2 \Theta}{2 m_{N} T} \cdot \exp \left(-\frac{A_{F} p_{\text {beam }}^{2} \sin ^{2} \Theta}{2 m_{N} T}\right)
$$

due to the fact that the probability distribution of transverse momenta obeys

$$
\begin{aligned}
\frac{1}{N} \frac{d N}{d p_{T}} & =\frac{p_{T}}{A_{F} m_{N} T} \cdot \exp \left(-\frac{p_{T}^{2}}{2 A_{F} m_{N} T}\right) \\
& =\frac{p_{T}}{\sigma_{p}^{2}} \cdot \exp \left(-\frac{p_{T}^{2}}{2 \sigma_{p}^{2}}\right)
\end{aligned}
$$

which is a Rayleigh distribution with the dispersion $\sigma_{p}=$ $\sqrt{A_{F} m_{N} T}$. The relativistic ideal gas model gives the probability distribution of emission angles for the projectile fragments to be

$$
\begin{aligned}
\frac{1}{N} \frac{d N}{d \Theta} & \\
= & \left(\sin 2 \Theta \cdot \exp \left(-\frac{A_{F}}{T} \sqrt{p_{\text {beam }}^{2} \sin ^{2} \Theta+m_{N}^{2}}\right)\right) \\
& \times\left(\int_{0}^{\pi} \sin 2 \Theta \cdot \exp \left(-\frac{A_{F}}{T} \sqrt{p_{\text {beam }}^{2} \sin ^{2} \Theta+m_{N}^{2}}\right) d \Theta\right)^{-1}
\end{aligned}
$$

due to the fact that the probability distribution of transverse momenta obeys

$$
\begin{aligned}
\frac{1}{N} \frac{d N}{d p_{T}} & \\
= & \left(p_{T} \cdot \exp \left(-\frac{1}{T} \sqrt{p_{T}^{2}+A_{F}^{2} m_{N}^{2}}\right)\right) \\
& \times\left(\int_{0}^{\infty} p_{T} \cdot \exp \left(-\frac{1}{T} \sqrt{p_{T}^{2}+A_{F}^{2} m_{N}^{2}}\right) d p_{T}\right)^{-1},
\end{aligned}
$$

which is the simplest Boltzmann distribution, where the chemical potential and quantum effects are neglected due to small values.

In high energy nucleus-nucleus collisions, we have $\sin \Theta \approx \Theta$ and $\sin 2 \Theta \approx 2 \Theta$ for the projectile fragments. Because the spectators are usually in a low excitation state, the classical ideal gas model can be used for the projectile fragment. Then, the $\Theta$ distribution of the projectile fragments can be approximately expressed by

$$
\begin{aligned}
\frac{1}{N} \frac{d N}{d \Theta} & \approx \frac{A_{F} p_{\text {beam }}^{2} \Theta}{m_{N} T} \cdot \exp \left(-\frac{A_{F} p_{\text {beam }}^{2} \Theta^{2}}{2 m_{N} T}\right) \\
& =\frac{\Theta}{\sigma_{\Theta}^{2}} \cdot \exp \left(-\frac{\Theta^{2}}{2 \sigma_{\Theta}^{2}}\right),
\end{aligned}
$$

which is a Rayleigh distribution with the dispersion $\sigma_{\Theta}=$ $\sqrt{m_{N} T /\left(A_{F} p_{\text {beam }}^{2}\right)}$.

In some cases, we have to use a two- or three-temperature picture to give descriptions for the projectile fragments [5]. The angular distribution is then the sum of two or three Rayleigh distributions with different weights and dispersions (temperatures), and the transverse momentum distribution is also the sum of two or three Rayleigh distributions with different weights and dispersions (temperatures). For the temperature fluctuations which appeared in the projectile fragment distributions, we can also use the Tsallis statistics to give descriptions.

\section{Conclusions}

From the above discussions, we obtain the following conclusions. 
(a) In the rest frame of an isotropic emission source, the spectra described by Tsallis, Boltzmann, Fermi-Dirac, Bose-Einstein, Tsallis form of Boltzmann, Tsallis form of Fermi-Dirac, and Tsallis form of Bose-Einstein distributions are compared in the present work.

(b) The quantum effect can be observed in the case of using the given set of parameters for Figures 1 and 2. At a given temperature, the three standard (Boltzmann, Fermi-Dirac, and Bose-Einstein) distributions result in narrow spectra, the Tsallis forms of the three standard distributions (Tsallis-B, Tsallis-FD, and Tsallis-BE distributions) result in wide spectra, and the Tsallis distribution results in between.

(c) For a given transverse momentum spectrum, the Bose-Einstein distribution shows higher temperature than that by the Boltzmann distribution (physical temperature), while other distributions show lower temperatures. The possible relations between the Boltzmann temperature and others are given by using the corrected factors.

(d) The related distributions can be also used in the descriptions of projectile fragments. Generally, the transverse momentum spectra and angular distributions of projectile fragments obey the Rayleigh distribution. One needs two or three Rayleigh distributions to describe the projectile fragment distributions. The temperature fluctuations which appeared in the projectile fragment distributions can be also described by the Tsallis statistics.

\section{Conflict of Interests}

The authors declare that there is no conflict of interests regarding the publication of this paper.

\section{Acknowledgments}

The authors acknowledge the referees and editor for their constructive comments. This work was partly finished at the State University of New York at Stony Brook, USA. One of the authors (Fu-Hu Liu) thanks Professor Dr. Roy A. Lacey and the members of the Nuclear Chemistry Group of Stony Brook University for their hospitality. The authors also acknowledge the supports of the National Natural Science Foundation of China (under Grant no. 10975095), the Open Research Subject of the Chinese Academy of Sciences LargeScale Scientific Facility (under Grant no. 2060205), the Shanxi Scholarship Council of China, and the Overseas Training Project for Teachers at Shanxi University.

\section{References}

[1] F.-H. Liu, "Particle production in Au-Au collisions at RHIC energies," Physics Letters B, vol. 583, no. 1-2, pp. 68-72, 2004.

[2] N. P. Andreeva, Z. V. Anzon, V. I. Bubnov et al., "Fragmentation of ${ }^{22} \mathrm{Ne}$ relativistic nuclei on photoemulsion nuclei," Soviet Journal of Nuclear Physics, vol. 47, no. 1, pp. 102-108, 1988.
[3] S. Kamel, "Transverse-momentum distribution of ultrarelativistic protons in electromagnetic dissociation of ${ }^{32} \mathrm{~S}$ into $\mathrm{p}+{ }^{31} \mathrm{P}$," Nuovo Cimento della Societa Italiana di Fisica A, vol. 112, no. 4, pp. 327-334, 1999.

[4] F.-H. Liu, "Pseudorapidity (rapidity) and transverse momentum distributions of negatively charged hadrons in high energy nucleus-nucleus collisions," Chinese Journal of Physics, vol. 38, no. 1, pp. 42-48, 2000.

[5] F.-H. Liu, "Emission of relativistic light fragments in nucleusemulsion collisions at high energy," Chinese Journal of Physics, vol. 38, no. 6, pp. 1063-1073, 2000.

[6] F.-H. Liu, "Transverse momentum distribution of strange particles in high-energy $\mathrm{Pb}-\mathrm{Pb}$ collisions," Chinese Journal of Physics, vol. 41, no. 4, pp. 357-371, 2003.

[7] K. B. Bhalla, M. Chaudhry, S. Lokanathan et al., "Relativistic $\alpha$-particles emitted in Fe-emulsion interactions at 1.7A GeV," Nuclear Physics A, vol. 367, no. 3, pp. 446-458, 1981.

[8] M. M. Aggarwal, K. B. Bhalla, G. Das, and P. L. Jain, "Angular distributions of relativistic alpha particles in heavy-ion collisions," Physical Review C, vol. 27, no. 2, pp. 640-649, 1983.

[9] P. Z. Ning, L. Li, and D. F. Min, Foundation of Nuclear Physics: Nucleons and Nuclei, Higher Education Press, Beijing, China, 2003.

[10] E. Q. Wang, H. R. Wei, M. A. Rahim, S. Fakhraddin, and F. H. Liu, "Multi-component Erlang distribution of final-state particles produced in high energy collisions," Indian Journal of Physics, vol. 87, no. 2, pp. 185-193, 2013.

[11] H.-R. Wei, Y.-H. Chen, L.-N. Gao, and F.-H. Liu, "Comparing multicomponent Erlang distribution and Lévy distribution of particle transverse momentum," Advances in High Energy Physics, vol. 2014, Article ID 782631, 16 pages, 2014.

[12] F.-H. Liu, Y.-Q. Gao, T. Tian, and B.-C. Li, “Transverse momentum and pseudorapidity distributions of charged particles and spatial shapes of interacting events in $\mathrm{Pb}-\mathrm{Pb}$ collisions at 2.76 TeV," Advances in High Energy Physics, Article ID 725739, 14 pages, 2014.

[13] C. Tsallis, "Possible generalization of Boltzmann-Gibbs statistics," Journal of Statistical Physics, vol. 52, no. 1-2, pp. 479-487, 1988.

[14] C. Tsallis, "Nonadditive entropy and nonextensive statistical mechanics-an overview after 20 years," Brazilian Journal of Physics, vol. 39, no. 2A, pp. 337-356, 2009.

[15] C. Tsallis, R. S. Mendes, and A. R. Plastino, "The role of constraints within generalized nonextensive statistics," Physica A, vol. 261, no. 3-4, pp. 534-554, 1998.

[16] W. M. Alberico and A. Lavagno, "Non-extensive statistical effects in high-energy collisions," The European Physical Journal A, vol. 40, no. 3, pp. 313-323, 2009.

[17] W. M. Alberico, P. Czerski, A. Lavagno, M. Nardi, and V. Somá, "Signals of non-extensive statistical mechanics in high-energy nuclear collisions," Physica A, vol. 387, no. 2-3, pp. 467-475, 2008.

[18] T. S. Biró, Is There a Temperature? Conceptual Challenges at High Energy, Acceleration and Complexity, vol. 171 of Fundamental Theories of Physics, Springer, Berlin, Germany, 2011.

[19] T. S. Biró, G. Purcsel, and K. Ürmössy, "Non-extensive approach to quark matter," The European Physical Journal A, vol. 40, no. 3, pp. 325-340, 2009.

[20] G. Wilk and Z. Włodarczyk, "Power laws in elementary and heavy-ion collisions : a story of fluctuations and nonextensivity?" The European Physical Journal A, vol. 40, no. 3, pp. 299-312, 2009. 
[21] G. Wilk and Z. Włodarczyk, "Multiplicity fluctuations due to the temperature fluctuations in high-energy nuclear collisions," Physical Review C, vol. 79, no. 5, Article ID 054903, 10 pages, 2009.

[22] B.-C. Li, Y.-Z. Wang, and F.-H. Liu, "Formulation of transverse mass distributions in $\mathrm{Au}-\mathrm{Au}$ collisions at $\sqrt{s_{N N}}=200$ GeV/nucleon," Physics Letters B, vol. 725, no. 4-5, pp. 352-356, 2013.

[23] A. Adare, S. Afanasiev, C. Aidala et al., "Identified charged hadron production in $p+p$ collisions at $\sqrt{s}=200$ and $62.4 \mathrm{GeV}$," Physical Review C, vol. 83, no. 6, Article ID 064903, 29 pages, 2011.

[24] B. I. Abelev, J. Adams, M. M. Aggarwal et al., "Strange particle production in $p+p$ collisions at $\sqrt{s}=200 \mathrm{GeV}$," Physical Review C, vol. 75, no. 6, Article ID 064901, 21 pages, 2007.

[25] K. Aamodt, N. Abel, U. Abeysekara et al., "Transverse momentum spectra of charged particles in proton-proton collisions at $\sqrt{s}=900 \mathrm{GeV}$ with ALICE at the LHC," Physics Letters B, vol. 693, no. 2, pp. 53-68, 2010.

[26] G. Aad, B. Abbott, J. Abdallah et al., "Charged-particle multiplicities in pp interactions measured with the ATLAS detector at the LHC," New Journal of Physics, vol. 13, no. 5, Article ID 053033, 68 pages, 2011.

[27] V. Khachatryan, A. M. Sirunyan, A. Tumasyan et al., "Transverse-momentum and pseudorapidity distributions of charged hadrons in pp collisions at $\sqrt{s}=0.9$ and $2.36 \mathrm{TeV}$,' Journal of High Energy Physics, vol. 2010, no. 2, Article ID 041, 35 pages, 2010.

[28] V. Khachatryann, A. M. Sirunyan, A. Tumasyan et al., "Transverse-momentum and pseudorapidity distributions of charged hadrons in $p p$ collisions at $\sqrt{s}=7 \mathrm{TeV}$," Physical Review Letters, vol. 105, no. 2, Article ID 022002, 14 pages, 2010.

[29] G. Wilk and Z. Włodarczyk, "Interpretation of the nonextensivity parameter q in some applications of Tsallis statistics and Levy distributions," Physical Review Letters, vol. 84, no. 13, Article ID $2770,2000$.

[30] J. Cleymans, G. I. Lykasov, A. S. Parvan, A. S. Sorin, O. V. Teryaev, and D. Worku, "Systematic properties of the Tsallis distribution: energy dependence of parameters in high energy p-p collisions," Physics Letters B, vol. 723, no. 4-5, pp. 351-354, 2013.

[31] J. Cleymans, "The Tsallis distribution for $p-p$ collisions at the LHC," Journal of Physics: Conference Series, vol. 455, Article ID 012049, 6 pages, 2013.

[32] M. D. Azmi and J. Cleymans, "Transverse momentum distributions in proton-proton collisions at LHC energies and Tsallis thermodynamics," Journal of Physics G: Nuclear and Particle Physics, vol. 41, no. 6, Article ID 065001, 2014.

[33] M. D. Azmi and J. Cleymans, "Transverse momentum distributions in $\mathrm{p}-\mathrm{Pb}$ collisions and Tsallis thermodynamics," http://arxiv.org/abs/1311.2909.

[34] C. Wong and G. Wilk, "Tsallis fits to $p_{T}$ spectra and multiple hard scattering in pp collisions at the LHC," Physical Review D, vol. 87, no. 11, Article ID 114007, 19 pages, 2013.

[35] C. Y. Wong and G. Wilk, "Tsallis fits to $\mathrm{p}_{T}$ spectra for $\mathrm{pp}$ collisions at the LHC," Acta Physica Polonica B, vol. 43, no. 11, pp. 2047-2054, 2012.

[36] B.-C. Li, Y.-Z. Wang, F.-H. Liu, X.-J. Wen, and Y.-E. Dong, "Particle production in relativistic $p p(\bar{p})$ and $A A$ collisions at
RHIC and LHC energies with Tsallis statistics using the twocylindrical multisource thermal model," Physical Review D, vol. 89, no. 5, Article ID 054014, 12 pages, 2014.

[37] F.-H. Liu, Y.-Q. Gao, and B.-C. Li, "Comparing two-Boltzmann distribution and Tsallis statistics of particle transverse momentums in collisions at LHC energies," The European Physical Journal A, vol. 50, no. 8, Article ID 123, 11 pages, 2014.

[38] J. Cleymans and D. Worku, "Relativistic thermodynamics: transverse momentum distributions in high-energy physics," European Physical Journal A, vol. 48, article 160, 2012.

[39] F. Büyükkiliç and D. Demirhan, "A fractal approach to entropy and distribution functions," Physics Letters A, vol. 181, no. 1, pp. 24-28, 1993.

[40] F. Pennini, A. Plastino, and A. R. Plastino, "Tsallis entropy and quantal distribution functions," Physics Letters. A, vol. 208, no. 4-6, pp. 309-314, 1995.

[41] J.-C. Chen, Z.-P. Zhang, G.-Z. Su, L.-X. Chen, and Y.-G. Shu, " $q$-generalized Bose-Einstein condensation based on Tsallis entropy," Physics Letters A, vol. 300, no. 1, pp. 65-70, 2002.

[42] A. M. Teweldeberhan, A. R. Plastino, and H. G. Miller, "On the cut-off prescriptions associated with power-law generalized thermostatistics," Physics Letters A, vol. 343, no. 1-3, pp. 71-78, 2005.

[43] J. M. Conroy and H. G. Miller, "Color superconductivity and Tsallis statistics," Physical Review D, vol. 78, no. 5, Article ID 054010, 5 pages, 2008.

[44] J. M. Conroy, H. G. Miller, and A. R. Plastino, “Thermodynamic consistency of the q-deformed Fermi-Dirac distribution in nonextensive thermostatics," Physics Letters A, vol. 374, no. 45, pp. 4581-4584, 2010.

[45] A. Lavagno, "Hot and dense hadronic matter in an effective mean-field approach," Physical Review C, vol. 81, no. 4, Article ID 044909, 2010.

[46] C. D. Dermer, "The production spectrum of a relativistic Maxwell-Boltzmann gas," The Astrophysical Journal, vol. 280, no. 1, part 1, pp. 328-333, 1984.

[47] F.-H. Liu, C.-X. Tian, M.-Y. Duan, and B.-C. Li, "Relativistic and quantum revisions of the multisource thermal model in highenergy collisions," Advances in High Energy Physics, vol. 2012, Article ID 287521, 9 pages, 2012.

[48] P. Braun-Munzinger, "Hadron production in nuclear collisions and the QCD phase boundary," Talk given at 45 years of nuclear theory at Stony Brook: a tribute to Gerald E. Brown, Stony Brook University, Stony Brook, NY, USA, (will be published as a special issue in Nuclear Physics A), 2013, http://tonic.physics.sunysb.edu/gerrybrown/program2.html.

[49] F.-H. Liu, Y.-H. Chen, H.-R. Wei, and B.-C. Li, "Transverse momentum distributions of final-state particles produced in soft excitation process in high energy collisions," Advances in High Energy Physics, vol. 2013, Article ID 965735, 15 pages, 2013.

[50] F.-H. Liu, T. Tian, and X.-J. Wen, "Transverse mass and rapidity distributions and space dispersion plots of (net-)protons in $\mathrm{Pb}$ $\mathrm{Pb}$ collisions at SPS energies," The European Physical Journal A, vol. 50, Article ID 50, 2014.

[51] F.-H. Liu, T. Tian, H. Zhao, and B.-C. Li, "Extracting chemical potentials of quarks from ratios of negatively/positively charged particles in high-energy collisions," The European Physical Journal A, vol. 50, no. 3, Article ID 62, 8 pages, 2014.

[52] B.-C. Li and L.-L. Wang, "Entropy in scalar $O(N)$ model with a spontaneously broken symmetry," International Journal of Modern Physics A, vol. 24, no. 30, pp. 5725-5736, 2009. 
[53] B.-C. Li and M. Huang, "Strongly coupled matter near phase transition," Journal of Physics G, vol. 36, no. 6, Article ID 064062, 5 pages, 2009.

[54] B.-C. Li, Y.-Y. Fu, L.-L. Wang, and F.-H. Liu, "Dependence of elliptic flows on transverse momentum and number of participants in $\mathrm{Au}+\mathrm{Au}$ collisions at $\sqrt{s N N}=200 \mathrm{GeV}$,' Journal of Physics G, vol. 40, no. 2, Article ID 025104, 9 pages, 2013. 

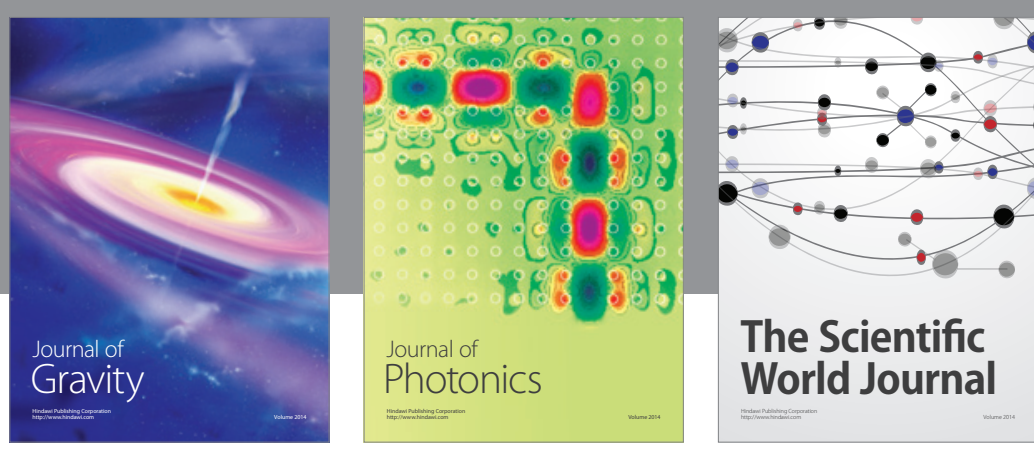

The Scientific World Journal
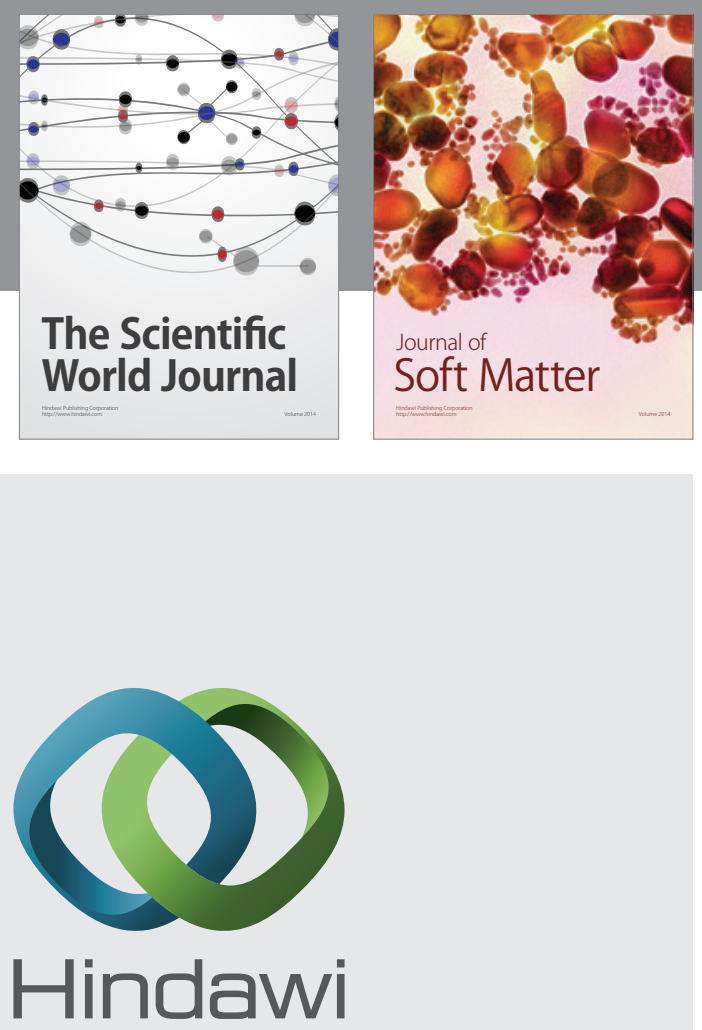

Submit your manuscripts at

http://www.hindawi.com

nternational Journal of

Statistical Mechanics
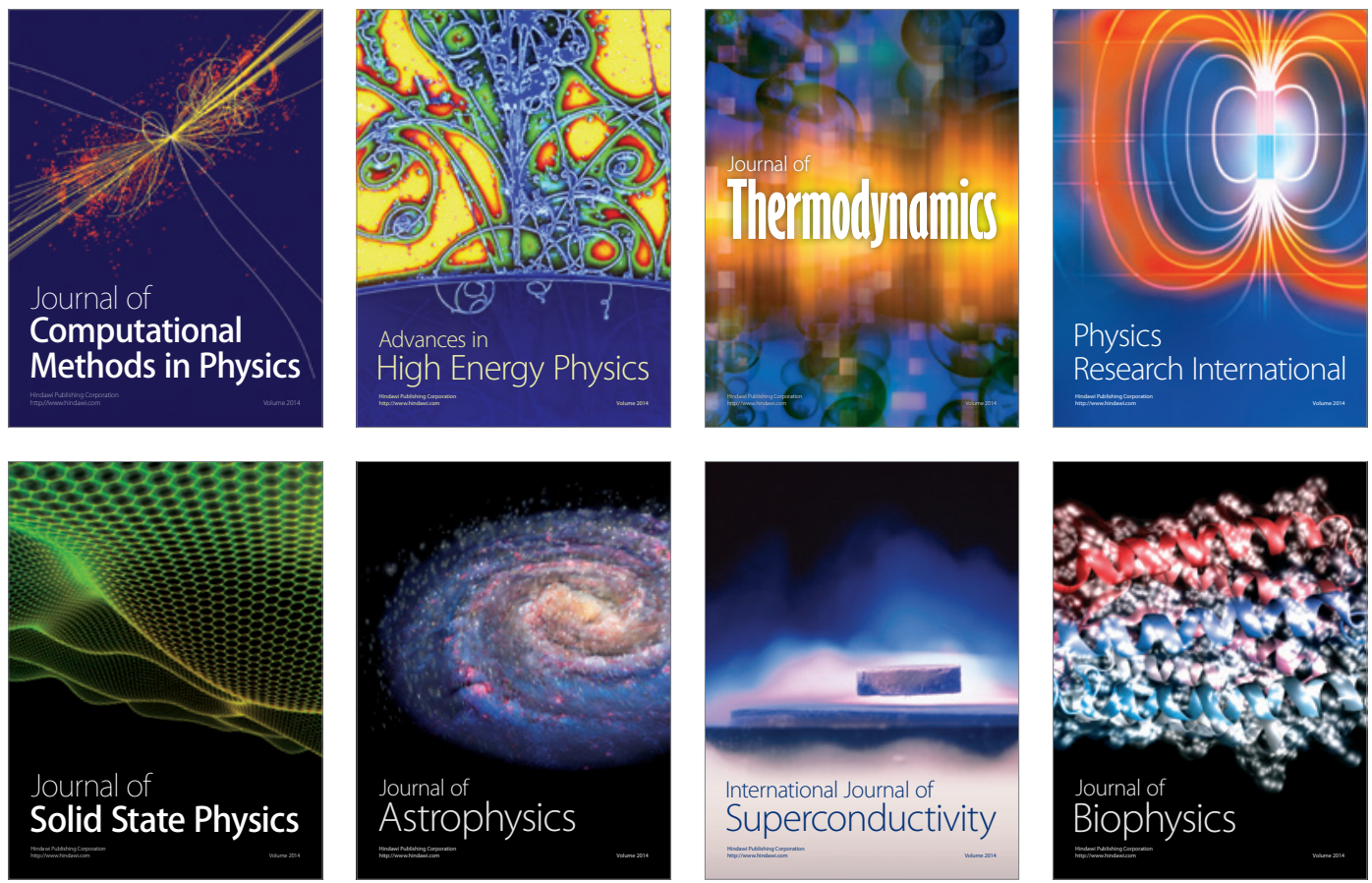
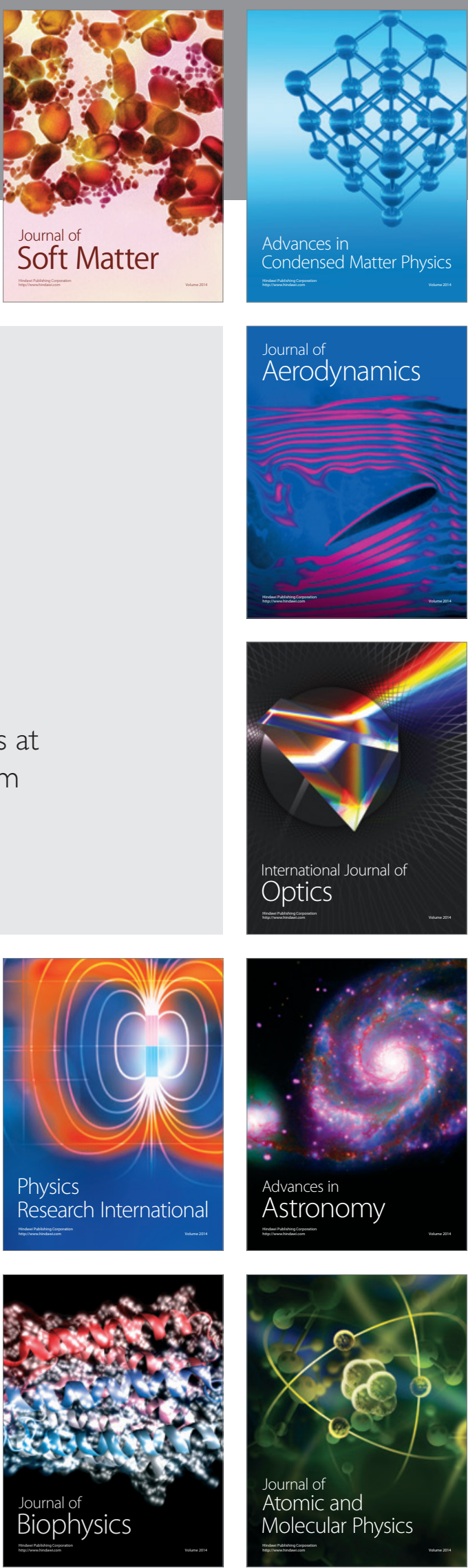